\title{
FERROMAGNETIC RESONANCE IN BULK-ACOUSTIC WAVE MULTIFERROIC DEVICES
}

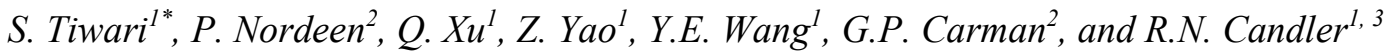 \\ ${ }^{1}$ Department of Electrical Engineering, University of California, Los Angeles, USA, \\ ${ }^{2}$ Department of Mechanical and Aerospace Engineering, University of California, Los Angeles, USA \\ ${ }^{3}$ California NanoSystems Institute, Los Angeles, USA
}

\begin{abstract}
The effect of ferromagnetic resonance (FMR) on the magnetomechanical coupling in thin-film multiferroic bulk-acoustic wave (BAW) devices is demonstrated. Devices consisting of piezoelectric and magneto-elastic materials are fabricated, and their electrical response is measured as a function of applied magnetic field magnitude and angle. As the applied magnetic field aligns the FMR frequency of the magneto-elastic layer with that of the BAW resonance of the structure, peaks in device conductance are observed. Approximate micro-magnetic simulations reproduce the trends seen experimentally. This represents the first investigation in the magneto-elastic coupling in thin-film BAW devices for potential applications for radio frequency (RF) miniature multiferroic devices.
\end{abstract}

\section{INTRODUCTION}

Recent interest in multiferroics, materials with coupled magnetic and electrical properties, has stimulated breakthroughs in a wide range of nanoscale magnetic systems, such as spin wave devices, non-volatile memory, and RF devices [1]. Multiferroics allow for the control of magnetization via electric fields, escaping the Ohmic losses of the typical electrical current control in miniature magnetic devices. However, single phase materials that exhibit these effects naturally are rare and have weak coupling. Strain-coupled multiferroic composites, laminates of piezoelectric and magnetostrictive materials which couple the electric and magnetic energy domains via mechanical strain, have demonstrated strong multiferroic coupling and offer a route for the realization of multiferroic devices.

To date, most multiferroic devices utilize a static magnetoelectric effect, where the DC magnetization is tuned using a DC voltage via strain-coupling [2]. This type of multiferroic coupling has been demonstrated for applications in spintronics, phase shifters, and memory devices. Relatively little work has been done on dynamic multiferroic coupling, where a time-varying voltage dynamically controls the magnetization state of a system via acoustic waves. Dynamic multiferroic coupling can also take advantage of FMR, greatly enhancing the coupling by allowing acoustic excitation of the magnetization at its resonance frequency. This enhancement of the coupling allows for RF applications in the $\mathrm{GHz}$ range.

Surface-acoustic wave (SAW) and BAW piezoelectric devices have already shown to be commercially successful at RF frequencies, finding applications in filters, duplexers, and oscillators [3]. This success make these devices very attractive candidates for dynamic multiferroics, where acoustic waves are necessary to mediate the magneto-electric coupling.

Previous work in this field has demonstrated SAW excitation of FMR in thin-film $\mathrm{Ni}[4,5]$. In these experiments, a set of interdigitated transducers (IDT's) excite a SAW on a $\mathrm{LiNbO}_{3}$ substrate. This SAW then travels across the surface through a $\mathrm{Ni}$ thin-film to be received by a second set of IDT's. The power received by the second set of IDT's is then measured as a function of an applied magnetic field magnitude and angle, showing maximum absorption at the FMR frequency and with a bias angle

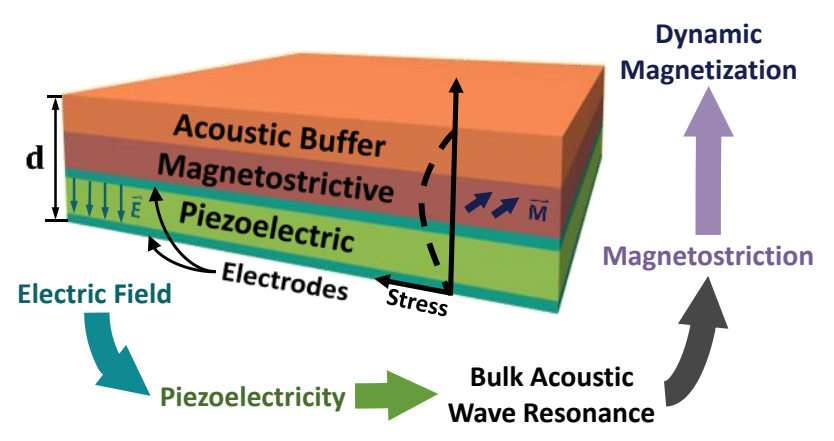

Figure 1: Structure of BAW multiferroic resonator. An applied electric field $(\vec{E})$ generates a standing acoustic wave. The standing acoustic wave leads to a dynamic excitation of the magnetic moment $(\vec{M})$ in the magnetostrictive layer. The total thickness (d) determines the resonant frequencies.

approximately $40^{\circ}$ to the propagation direction of the SAW.

FMR has also been shown to allow for tuning in high-overtone BAW devices [6]. In these devices zinc oxide ( $\mathrm{ZnO})$ with a tilted caxis is deposited on an epitaxial yttrium iron garnet (YIG) magnetic film on a gadolinium gallium garnet (GGG) substrate. When electrically excited, the piezoelectric $\mathrm{ZnO}$ creates both shear and longitudinal standing acoustic waves throughout the GGG substrate, YIG, and $\mathrm{ZnO}$. The effect of an applied bias on the reflection coefficient, $S_{11}$, was then measured. It was found that the resonance frequency could be tuned by $0.25 \mathrm{MHz}$ around $2 \mathrm{GHz}$ due to the magneto-elastic interaction.

In this work we investigate the effect of FMR in thin-film BAW resonant devices (Figure 1). Unlike the prior work, these devices do not require thick magnetic or piezoelectric substrates and can be fabricated directly on Si. As the resonance is completely contained within the thin-film structure, substrate isolation and attenuation of the acoustic wave does not need to be considered. In addition to the fundamental thickness-extensional mode, these devices can take advantage of any available low order harmonic modes to excite FMR in the magnetoelastic layer, avoiding the complications involved when using higher order harmonic devices. Such a structure has previously been proposed as a miniature antenna, potentially allowing for highly efficient radiation [7]. We also present the first experimental study on the dependence of outof-plane angular dependence of the magnetic bias on the magnetoelastic response in thin-films.

\section{THEORY} given by

The energy density due to the magneto-elastic interaction is

$$
u_{m e}=-\frac{3}{2} \lambda_{s} \sigma\left(\cos ^{2} \theta-\frac{1}{3}\right)
$$

where $\sigma$ is the applied stress, $\lambda_{s}$ is the saturation magnetostriction, $\theta$ is the angle between the applied stress and the net magnetization. 


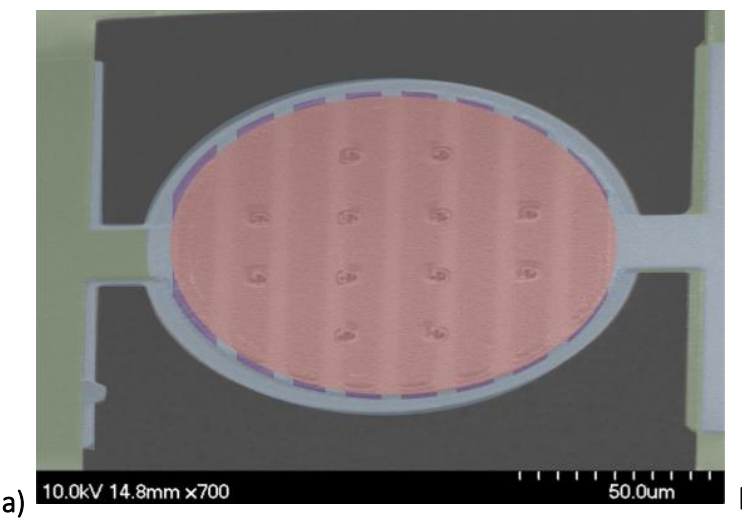

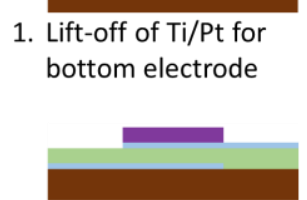

4. Lift-off of $\mathrm{Ni}$
2. Reactive sputtering of AIN

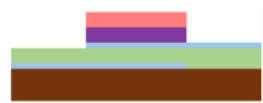

5. Deposition and etching of SiN

\begin{tabular}{|c|c|}
\hline \multicolumn{2}{|c|}{ Legend } \\
Silicon & Niatinum \\
\hline $\begin{array}{c}\text { Aluminum } \\
\text { Nitride }\end{array}$ & $\begin{array}{c}\text { Silicon } \\
\text { Nitride }\end{array}$ \\
\hline
\end{tabular}

1. Lift-off of Ti/Pt for top electrode

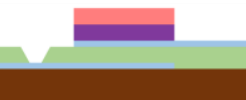

6. Etching of AIN

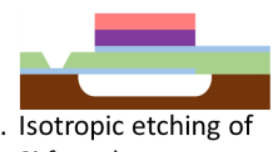

Si for release

Figure 2: a) Colorized scanning electron microscope (SEM) image of a completed device. b) Manufacturing process for device fabrication.

If it is assumed that a normal stress is applied to the z-axis, the effective magnetic field due to an applied stress can be calculated as follows:

$$
\vec{h}_{m e}=-\frac{1}{\mu_{0}} \frac{d u_{m e}}{d M_{z}} \hat{z}=\frac{1}{\mu_{0}} \frac{3 \lambda_{s}}{M_{s}^{2}} M_{Z} \sigma \hat{z}
$$

Here $M_{S}$ is the saturation magnetization and $M_{z}=M_{s} \cos \theta$ is the magnetization along the axis of the applied stress.

In the static case, the magnetization of the magnetic material will rotate in the direction of the effective field until it minimizes the total energy that it experiences. In the dynamic case however, the precession of the magnetization around the DC magnetic bias field cannot be ignored and the Landau-Lifshitz-Gilbert equation must be used to describe the magnetization dynamics. In the absence of dissipation, this equation is

$$
\frac{d}{d t}(\vec{M}+\vec{m})=-\mu_{0} \gamma(\vec{M}+\vec{m}) \times\left(\vec{H}_{0}+\vec{H}_{d}+\vec{h}_{d}+\vec{h}_{m e}\right)
$$

where $\vec{M}$ is the DC magnetization, $\vec{m}$ is the oscillating magnetization, $\gamma$ is the gyromagnetic ratio, $\vec{H}_{0}$ is the applied bias field, $\vec{H}_{d}$ is the demagnetization field due to DC magnetization, and $\vec{h}_{d}$ is the demagnetization field due to the oscillating magnetization.

This equation can be solved to give the small-signal magnetic susceptibility tensor $(\chi)$ in the frequency domain, and is found to be a function of the angle of DC magnetization and the magnitude of the applied field [8]. It can be shown using this calculation that the magnitude of the susceptibility exhibits resonant behavior, increasing dramatically at frequencies near its resonant frequency. The resonant frequency, known as the FMR frequency, can be calculated from the Kittel equation, which in the case of a thin-film with an in plane magnetic bias is

$$
\omega_{r}=\mu_{0} \gamma \sqrt{\left(H_{0}+M_{s}\right) H_{0}}
$$

The susceptibility tensor can be used with the effective magnetoelastic magnetic field to find piezomagnetic coefficients analogous to those of piezoelectric materials [7].

$$
\vec{m}=\chi \vec{h}_{m e}=\left(\chi \cdot \frac{1}{\mu_{0}} \frac{3 \lambda_{s}}{M_{s}^{2}} M_{z} \hat{z}\right) \sigma_{33}=d_{i 3} \sigma_{33}
$$

Just as piezomagnetic coefficients can be defined, a magnetomechanical coupling coefficient can similarly be defined.

$$
K_{33}^{2}=\frac{d_{3 i}^{t} \cdot \mu_{i j}^{-1} \cdot d_{j 3}}{c_{33}}=\frac{\left(\left(\chi_{3 i} \frac{13 \lambda_{S}}{\mu_{0} M_{S}^{2}} M_{z}\right)^{t} \cdot(1+\chi)_{i j}^{-1} \cdot\left(\chi_{j 3} \frac{13 \lambda_{S}}{\mu_{0} M_{S}^{2}} M_{z}\right)\right)}{\mu_{0} c_{33}} \propto \chi
$$

Here $c_{33}$ is the out-of-plane stiffness of the magneto-elastic material, $t$ is the transpose operator, $\mu=\mu_{0}(1+\chi)$ is the permeability, and $i$ and $j$ are the tensor indices. Since the susceptibility is a function of frequency, this equation shows that the magneto-elastic coupling is highly dependent on frequency, with the magneto-mechanical coupling coefficient approaching its peak near FMR. The magnetization naturally wants to oscillate at the FMR frequency, which means that the magnetization couples best with stimuli that oscillate at the FMR frequency (regardless of whether it's a magnetic field or strain). This effect has previously been demonstrated both numerically and experimentally $[5,9]$.

\section{EXPERIMENTAL DETAIL}

\section{Design and Fabrication}

To experimentally investigate this coupling, a tri-layer structure made of a piezoelectric layer (720 nm AlN), a magnetoelastic layer $(735 \mathrm{~nm} \mathrm{Ni})$, and an acoustic buffer layer $(300 \mathrm{~nm}$ $\mathrm{Si}_{3} \mathrm{~N}_{4}$ ) were fabricated (Figure 2). An AC voltage applied across the piezoelectric generates an acoustic standing wave throughout the structure. The strain from this standing wave then drives the oscillation of the magnetization in the magneto-elastic layer. The acoustic buffer ensures that the peak of the strain wave is in the

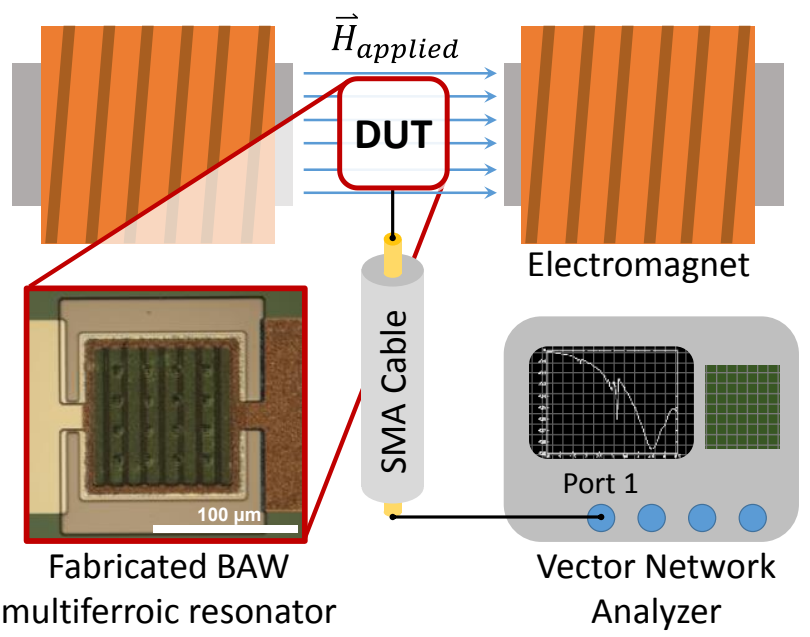

Figure 3: Diagram measurement system for magneto-mechanical coupling experiments. 

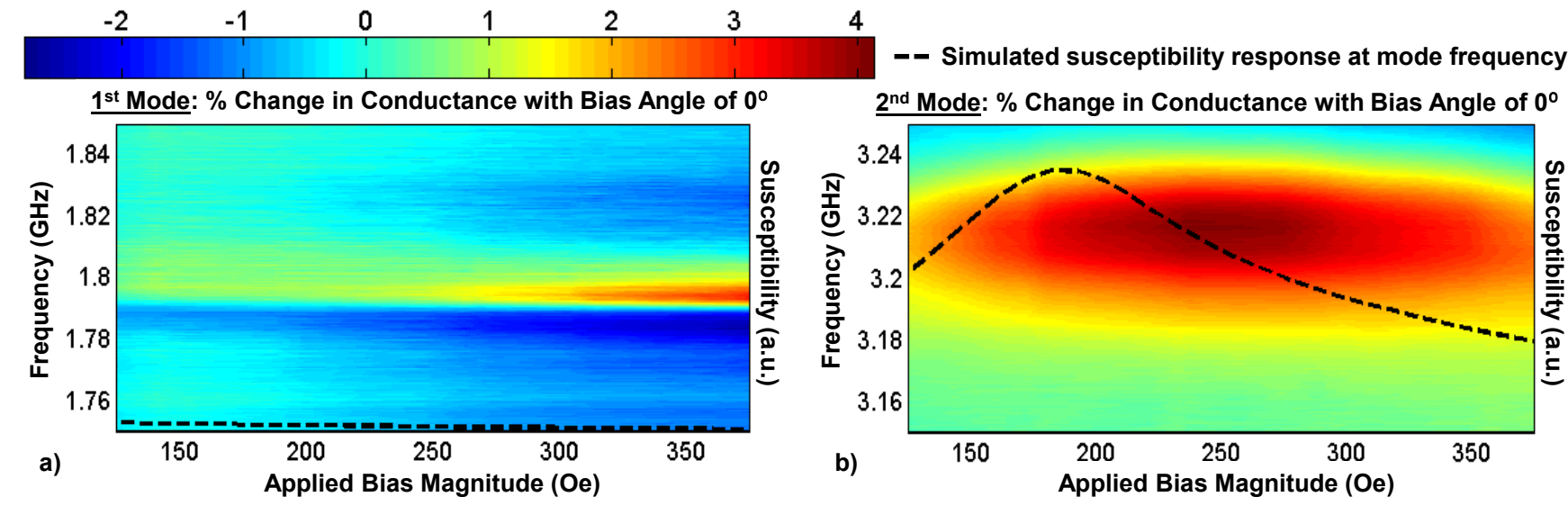

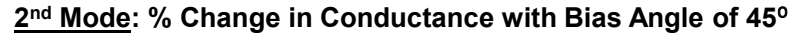

2nd Mode: \% Change in Conductance with Bias Angle of $85^{\circ}$
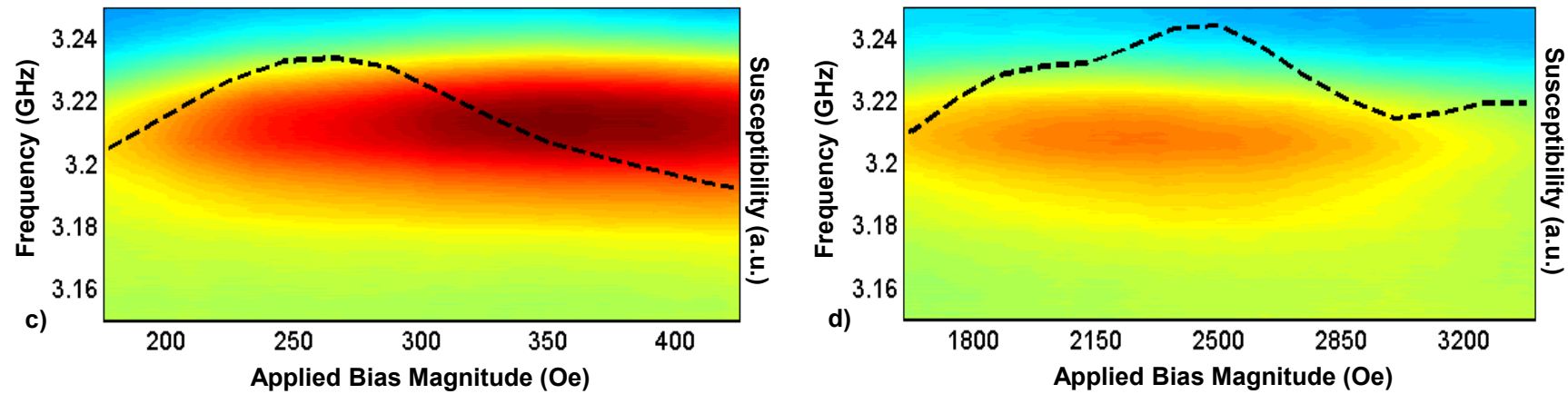

Figure 4: Colormaps of the percent change of device conductance as a function of frequency and magnetic bias. Red indicates an increase of conductance and blue indicates a decrease in conductance. Dashed lines are of the estimated FMR response found through simulation. a) Effect of magnetic field at the first resonant mode $\left(1.8 \mathrm{GHz}\right.$ ) and $0^{\circ}$ bias angle. b) Effect of magnetic field at the second resonant mode $(3.2 \mathrm{GHz})$ and $0^{\circ}$ bias angle. c) Effect of second resonant mode at $45^{\circ}$ bias angle. d) Effect of second resonant mode at $85^{\circ}$ bias angle.

magnetostrictive layer to maximize the coupling of mechanical energy to magnetic energy. Thicknesses were chosen such that the peak of the acoustic wave would lie in the center of the Ni layer. Gaps were included in the magneto-elastic layer in an attempt to minimize the eddy current loss associated with a continuous film.

First, $10 \mathrm{~nm} \mathrm{Ti} / 100 \mathrm{~nm} \mathrm{Pt}$ is evaporated on high resistivity Si $(>10 \mathrm{k} \Omega \cdot \mathrm{cm})$ to serve as the bottom electrode for the device and patterned via a lift-off process. The choice of $\mathrm{Pt}$ as the bottom electrode is integral for device operation as it promotes growth of high quality c-axis oriented AlN. Next, $720 \mathrm{~nm}$ of AlN blanket film is reactively sputtered on the wafer. $10 \mathrm{~nm} \mathrm{Ti} / 100 \mathrm{~nm} \mathrm{Pt}$ is then evaporated and lifted-off to pattern the top electrode layer. Sputter deposition of $10 \mathrm{~nm} \mathrm{Ti} / 735 \mathrm{~nm} \mathrm{Ni}$ follows and is also patterned by a lift-off process. The pressure for the Ni deposition was tuned to minimize residual film stress and to avoid the "winging" effects typical of sputter lift-off process by keeping the mean free path of the sputtered Ni long. Following Ni deposition, $530 \mathrm{~nm}$ of $\mathrm{Si}_{3} \mathrm{~N}_{4}$ is deposited via PECVD and then etched in $\mathrm{CHF}_{3}$ plasma. The AlN layer is then etched in a mixture of $\mathrm{CF}_{4}$ and Ar plasma for access to the substrate and bottom electrode. This etch has high selectivity to $\mathrm{Si}$ with respect to $\mathrm{Pt}$, meaning that over-etching will etch into the substrate and allow for an easier release without damaging the $\mathrm{Pt}$ bottom electrode. The devices are then released from the substrate using $\mathrm{XeF}_{2}$ gas phase etching of the $\mathrm{Si}$. During this step, the $\mathrm{Si}_{3} \mathrm{~N}_{4}$ was unintentionally etched to a thickness of approximately $300 \mathrm{~nm}$.

\section{Measurement}

To facilitate measurement, a fabricated device was packaged as the terminating load of a coplanar waveguide (CPW) transmission line, which connects to a vector-network analyzer (VNA) via a flange mount SMA connector (Figure 3). The packaged device was then placed in an electromagnet where its reflection coefficient, $\mathrm{S}_{11}$, could be measured as a function of the applied magnetic field magnitude and angle. Control of the applied angle was made possible through the use of $3 \mathrm{D}$ printed mounts. Measurements were made at angles of $0^{\circ}, 45^{\circ}$, and $85^{\circ}$ relative to the plane of the device. From the measured $S_{11}$ values, the admittance of the packaged system was then calculated to study the electrical response of the system as a function of the applied magnetic field.

\section{RESULTS}

From the measured $\mathrm{S}_{11}$ response, the complex admittance of the system was calculated. The system conductance, which is related to the amount of power dissipated by the system, is then found by taking the real part of the admittance. As the magnetic field increases, the ferromagnetic resonance frequency of the Ni film also increases, as seen by the Kittel equation (Equation 4). The oscillation of the magnetization is maximized when the FMR frequency aligns with an acoustic resonance mode, causing the most power to be absorbed by the magneto-elastic layer. This manifests itself as a peak in the measured conductance.

Figure 4 shows colormaps of the percent change in conductance of the packaged system as a function of magnetic bias magnitude and frequency at the first two resonant modes. The percent change is measured relative to the frequency response at 
approximately zero magnetic bias, perfect zero bias being unachievable due to nonzero magnetic remanence of the electromagnet poles. At $1.8 \mathrm{GHz}$, the fundamental resonant mode, no field dependence of the conductance is seen as the bias needed for FMR is too low to align the magnetic moments, so FMR is not possible. The changes in conductance seen here at high bias were determined to be due to trace magnetic material in the SMA connectors and cables. Effects can be seen at $3.2 \mathrm{GHz}$, the second harmonic of mechanical resonance, under the same bias fields, confirming the frequency dependence of the magneto-mechanical coupling. Here the highest change was found at an applied magnetic bias of $237 \mathrm{Oe}$, indicating that this bias stimulates FMR at $3.2 \mathrm{GHz}$.

When the bias moves out-of-plane a higher magnetic field is needed to stimulate FMR due to the increase in demagnetization energy. At $45^{\circ}$, the peak conductance at $3.2 \mathrm{GHz}$ was found to be at 361 Oe. At $85^{\circ}$ a much higher field is needed to magnetize the film in the mostly out-of-plane orientation, with the peak conductance occurring at a bias of 2320 Oe.

To confirm that this effect is due to FMR, micro-magnetic simulations were done in the Object Oriented MicroMagnetic Framework (OOMMF) developed by NIST to estimate the magnetic susceptibility of the film [10]. A $735 \mathrm{~nm}$ Ni thin film is stimulated by an RF magnetic field at the resonant frequency of the mode of interest. The magnitude of the applied DC magnetic bias field is then stepped through values within the range applied during measurement. The amplitude of the steady-state magnetization oscillation is calculated as a function of magnetic field. This corresponds to the dashed lines in Figure 4. Maxima in these curves indicate a bias that stimulates FMR. The bias fields corresponding to the maximum change in conductance follow the same trends of the FMR response estimated by the OOMMF model and match the simulated values within $30 \%$ or less. Discrepancies between measurement and modelling are expected as crystallographic, domain, and exchange effects are not included in the model. Exchange effects, in particular, have been found to be important when investigating the magneto-elastic response near FMR [11]. However, the matching trends of maximum conductance and simulated susceptibility with respect to bias angle strongly suggests that this effect is due to FMR within the magneto-elastic layer.

\section{CONCLUSION}

In this work we investigate the effect of FMR on the magnetoelastic coupling of thin-film BAW devices. We find that the device conductance peaks at particular magnetic bias magnitudes and the trends of the angular dependence match that of FMR simulated with OOMMF. With further advancements of the experimental system, full analysis of power dissipation due to FMR will enable the possibility of quantitative measurements of dynamic multiferroic coupling for applications to RF devices.

\section{ACKLNOWLEDGEMENTS}

The authors would like to thank Dr. Gianluca Piazza and Dr. Jeronimo Segovia for their assistance with the deposition of aluminum nitride. The authors would also like to thank the UCLA Nanoelectronics Research Facility and the Integrated Systems Nanofabrication Cleanroom for use of their extensive fabrication facilities. This work was supported by the National Science Foundation through the Cooperative Agreement Award EEC1160504 for Solicitation NSF 11-537 (TANMS) managed by Dr. Deborah J. Jackson.

\section{REFERENCES}

[1] R. Ramesh, and N.A. Spaldin. "Multiferroics: progress and prospects in thin films." Nature materials 6.1 (2007) pp. 21-29.

[2] M. Liu, N.X. Sun. "Voltage control of magnetism in multiferroic heterostructures." Philosophical Transactions of the Royal Society of London A: Mathematical, Physical and Engineering Sciences 372.2009 (2014): 20120439.

[3] R. Aigner, "SAW and BAW technologies for RF filter applications: A review of the relative strengths and weaknesses." Ultrasonics Symposium, 2008. IUS 2008. IEEE. IEEE (2008).

[4] L. Dreher, et al. "Surface acoustic wave driven ferromagnetic resonance in nickel thin films: Theory and experiment." Physical Review B 86.13 (2012): 134415.

[5] D. Labanowski, A. Jung, and S. Salahuddin. "Power absorption in acoustically driven ferromagnetic resonance." Applied Physics Letters 108.2 (2016): 022905.

[6] S. Alekseev, et al. "Tunable HBAR based on magnetoelectric YIG/ZnO structure." Ultrasonics Symposium (IUS), 2012 IEEE International. IEEE (2012)

[7] Z. Yao, et al. "Bulk Acoustic Wave-Mediated Multiferroic Antennas: Architecture and Performance Bound." Antennas and Propagation, IEEE Transactions on 63.8 (2015): pp. 33353344.

[8] D.M. Pozar, Microwave Engineering, John Wiley \& Sons, Inc., New Jersey, 2012.

[9] Z. Yao, and Y.E. Wang. "Bulk acoutic wave mediated multiferroic antennas near ferromagnetic resonance." Antennas and Propagation \& USNC/URSI National Radio Science Meeting, 2015 IEEE International Symposium on. IEEE (2015).

[10] M.J. Donahue, and D.G. Porter. "OOMMF User's Guide, Version 1.0, Interagency Report NISTIR 6376, National Institute of Standard and Technology, Gaithersburg, MD, 1999." URL: http://math. nist. gov/oommf (2010).

[11] P.G. Gowtham, et al. "Traveling surface spin-wave resonance spectroscopy using surface acoustic waves." Journal of Applied Physics118.23 (2015): 233910.

CONTACT

*S. Tiwari, tel: +1-951-236-5952; stiwari@ucla.edu 